\title{
All-Solid-Sate pH sensing material based on cysteic acid/graphene oxide nanocomposites
}

\author{
Lin Liu ${ }^{\mathrm{a}}$, Yuting Huang ${ }^{\mathrm{b}}$, Jing Zhao ${ }^{\mathrm{a}}$, Zhijie Zhuc ${ }^{\mathrm{c}}$, Huaihao Zhang ${ }^{\mathrm{a}}$, Chengyin Wang ${ }^{\mathrm{a}, *}$ \\ ${ }^{a}$ College of Chemistry and Chemical Engineering, Jiangsu Key Laboratory of environmental engineering and monitoring, \\ Yangzhou University, 180 Si-Wang-Ting Road, Yangzhou 225002, China. \\ ${ }^{\mathrm{b}}$ School of Environmental Science and Technology, Dalian University of Technology, 2 Liao-Ning Road, Dalian, 116024, \\ China. \\ ${ }^{\mathrm{c}}$ Yangzhou Educational Service Centre, 36 Jin-Zhong Road, Yangzhou, 225002, China.
}

*Corresponding Author: wangcy@yzu.edu.cn

\begin{abstract}
Novel cysteic acid/graphene oxide composite film on glassy carbon electrode has been developed through the electrochemical oxidation of $L$-cysteine (CySH) for constructing an all-solid-state $\mathrm{pH}$ sensor. Potentiometric $\mathrm{pH}$ response of the cysteic acid/graphene oxide modified electrode in Britton-Robinson buffers revealed a linear working range from $\mathrm{pH} 2$ to12 with a slope of about -50.2 $\mathrm{mV} \cdot \mathrm{pH}^{-1}$ and fast response (less than 1 minute). The as-developed $\mathrm{pH}$ sensor also showed excellent reproducibility and high stability and had been successfully used to detect real samples with satisfactory results.
\end{abstract}

Keywords: graphene oxide, cysteic acid, $L$-cysteine, solid-state $\mathrm{pH}$ sensor.

\section{Introduction}

Graphene oxide (GO) was regarded as a two-dimensional-layer precursor for synthesizing graphene derivatives ${ }^{(1)}$. GO containing various oxygen groups, such as epoxy, hydroxyl, and carboxyl groups can be easily dispersed in common organic solvents, which enables GO to form stable suspensions with biomolecules, polymers and solvents, and nanocomposite films ${ }^{(2)}$. These reactive oxygen groups also offer good active sites for functionalizing $\mathrm{GO}$ and immobilizing electroactive species ${ }^{(3)}$. Owing to its excellent electronic, thermal and mechanical properties ${ }^{(4)}$, GO has been widely applied in the electrochemical sensor $^{(5)}$, nanoelectronics ${ }^{(6)}$, lithium battery $^{(7)}$ and supercapacitor ${ }^{(8)}$. In recent years, many studies have been focusing on preparation GO composite materials to construct electrochemical sensors. $L$-cysteine is known as an important amino acid for several biological equilibriums. It has been reported that cysteic acid can be obtained through electrochemically oxidizing $L$-cysteine ${ }^{(9)}$. Cysteic acid with carboxylate and sulfonated groups is referred to as a steady and biocompatible material for the fabrication sensors. Therefore, the cysteic acid/GO composite film would exhibit good mechanical properties and chemical reactivity owing to various functional groups for sensor applications.

As for $\mathrm{pH}$ value measurement, it is very important in many fields, such as food industries, environment monitoring, farming geoponics, clinical and biological sciences ${ }^{(10)}$. It is crucial to choose a reliable method to analyze the acidity of aqueous solution. Although the traditional glass bulb $\mathrm{pH}$ electrode is a well established tool in the measurement of $\mathrm{pH}$, it has a lot of disadvantages which cannot be avoided, such as mechanical fragility, instability, high-cost, high-resistance, special treatment before and after use ${ }^{(11)}$. It can't be applied in some special applications, such as high-pressure, high-temperature or high-viscosity fluid environment. Since the use of a glass membrane electrode to determine the hydrogen ion concentration in aqueous solutions is frequent and necessary, great efforts has been made to develop all-solid $\mathrm{pH}$ sensors to avoid these drawbacks, and a variety of alternatives have been demonstrated. New techniques and methods have broadened the scope of $\mathrm{pH}$ detection. For examples, metal/metal oxide ${ }^{(12,13)}$, ion sensitive field-effect transistors (ISFET) ${ }^{(14,15)}$, fiber-optical techniques ${ }^{(16,17)}$, and 
sensing thin film materials have been extensively explored to fabricate the all-solid $\mathrm{pH}$ sensors.

In this paper, we fabricated a novel solid-state $\mathrm{pH}$ sensor based on cysteic acid/GO composite film. The cysteic acid was obtained from electrochemical oxidation of $L$-cysteine $(\mathrm{CySH})$ on the surface of GO modified electrode. Owing to carboxylate and sulfonated groups in cysteic acid and $\mathrm{GO}$ with various oxygen containing functional groups, the proposed $\mathrm{pH}$ sensor achieved excellent electromotive force (emf) response with a wide linear range from $\mathrm{pH} 2$ to12 at $25{ }^{\circ} \mathrm{C}$ and high stability. We had successfully employed this proposed $\mathrm{pH}$ sensor to detect real samples and obtained satisfactory results. This novel GO/cysteic acid composite film could have great potential in various analytic applications.

\section{Experimental}

\subsection{Chemicals and apparatus}

SP-1 natural graphite powders were obtained from Bay Carbon, MI, USA. L-cysteine (CySH) was purchased from Sigma-Aldrich Co., Ltd. Phosphoric acid, acetic acid, boric acid, sodium hydroxide and other reagents used in this experiment were all of analytical grade. All solutions were prepared with deionized water.

Cyclic voltammetry (CV) experiment was carried out on a CHI 660a electrochemical workstation (Shanghai Chenhua, China). We employed a conventional three-electrode system with a GO modified glassy carbon electrode (3.0 $\mathrm{mm}$ in diameter) as a working electrode, a platinum wire as an auxiliary electrode, and a saturated calomel electrode (with $2.0 \mathrm{~mol} / \mathrm{L} \mathrm{KNO}_{3}$ salt bridge) as a reference electrode. Open-circuit potential (OCP) of the cysteic acid/GO modified electrode was measured as a function of $\mathrm{pH}$ value of the sample solutions by using $\mathrm{CHI}$ 660 a electrochemical workstation. Field emission scanning electron microscope (FESEM) images were captured by S-4800 II FESEM (Hitachi High-Technologies Corporation, Japan) at an accelerating voltage of $15.0 \mathrm{kV}$.

\subsection{Fabrication of cysteic acid/graphene oxide composite films}

Firstly, the glassy carbon electrodes were polished with $1 \mu \mathrm{m}$ and $0.05 \mu \mathrm{m} \alpha$-alumina powders respectively, and then sonicated in 1:1 nitric acid, acetone and de-ionized water successively. Natural graphite powders were oxidized to GO using a modified Hummers method ${ }^{(18)}$. Graphene oxide suspension was prepared by dispersing $3.0 \mathrm{mg} \mathrm{GO}$ in $20 \mathrm{~mL}$ THF under ultrasonication for 30 minutes. A $10 \mu \mathrm{L}$ aliquot of the suspension was dropped directly on glassy carbon electrode surface and had it dried at the room temperature, and then obtained GO modified electrode. The cysteic acid/GO composite film electrodes were prepared by cycle scanning the electrodes between $-0.8 \mathrm{~V}$ and +2.2 $\mathrm{V}$ (vs. SCE) at a scanning rate of $200 \mathrm{mV} \cdot \mathrm{s}^{-1}$ in $0.04 \mathrm{M}$ $\mathrm{HCl}$ solution containing $2.5 \times 10^{-3} \mathrm{M} L-\mathrm{Cysteine}(\mathrm{CySH})$ for 10 consecutive cycles, and the result was shown in Fig. 1. The SEM image of the as-obtained cysteic acid/GO composite film was shown in Fig. 2. The modified electrode was then electroactivated by cyclic scanning from $0 \mathrm{~V}$ to $+1.5 \mathrm{~V}$ in the $0.5 \mathrm{M} \mathrm{H}_{2} \mathrm{SO}_{4}$ solution until a steady cyclic voltammogram was obtained.

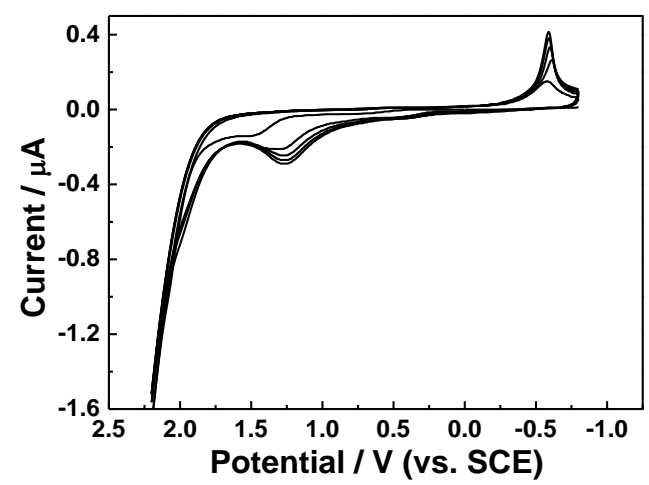

Fig. 1. Cyclic voltammograms of the GO/GCE in $0.04 \mathrm{M}$ $\mathrm{HCl}$ solution containing $2.5 \times 10^{-3} \mathrm{M}$ L-cysteine $(\mathrm{CySH})$. Scan rate: $200 \mathrm{mV} \cdot \mathrm{s}^{-1}$; Scan potential: -0.8 to $+2.2 \mathrm{~V}$; Consecutive cycle: 10 .

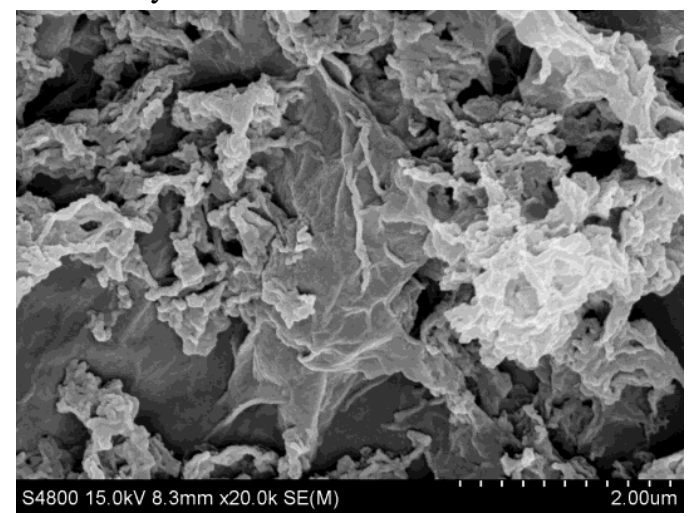

Fig. 2. SEM image of cysteic acid/GO composite film.

\section{Results and discussions}

3.1 Electrochemical oxidation of L-cysteine and the mechanism of cysteic acid/graphene oxide for $\mathbf{p H}$ sensing

Electrochemical oxidation of $\mathrm{CySH}$ on GO/GCE was 
investigated by the cyclic voltammetry. $\mathrm{CySH}$ can be adsorbed on the electrode by using voltammetric measurement or under high positive potential on the GCE electrode and further oxidated to cysteic acid ${ }^{(19)}$. A typical cyclic voltammogram from Fig. 1 shows that there is an irreversible oxidation peak at ca. $+0.8 \mathrm{~V}$, which implies that $\mathrm{CySH}$ is oxidized to $L$-cystine (CySSCy). And there is another irreversible oxidation peak at $1.3 \mathrm{~V}$ corresponding to the generation of $\mathrm{CySO}_{3} \mathrm{H}$. The detailed mechanism had been investigated in our previous study ${ }^{(20)}$. GO are functionalized with oxygen containing functional groups proved to be effective to improve interfacial bonding between polymer and GO. Furthermore, the functional group $-\mathrm{SO}_{3} \mathrm{H}$ of cysteic acid was confirmed to be strongly adsorbed at electrode through cyclic voltammetric and polarization measurements ${ }^{(21)}$. Therefore, the as-obtained cysteic acid/GO exhibited great stability and had good chemical reactivity owing to various functional groups.

Cysteic acid with carboxylate and sulfonated groups can be used as an electrode modifier due to its attractive ion-exchange characteristics. In addition, GO with a lot oxygen containing functional groups such as carboxyl, hydroxyl and phenol would be attributed to the good $\mathrm{pH}$ sensing. The cysteic acid/GO composite film had possessed the advantages of the above two materials and achieved excellent $\mathrm{pH}$ sensing performance.

\subsection{Selection of the optimal operation conditions}

The $\mathrm{pH}$ response of the cysteic acid/GO was affected by the amount of the GO loaded on the electrode surface. The same volume $(10 \mu \mathrm{L})$ of the suspensions with different concentrations $\left(0.05,0.1,0.15,0.20,0.25 \mathrm{mg} \cdot \mathrm{mL}^{-1}\right)$ of the GO was used to seek the optimal amount of GO during the preparation process. After the cysteic acid film was formed on the GO/GCE electrode by electroxidation of $\mathrm{CySH}$, the potentiometric responses of the $\mathrm{pH}$ sensors based on as-obtained composite film for the detection $\mathrm{pH}$ values from 2.0 to 12.0 was recorded. With the increment of the GO concentration, the potentiometric response increased accordingly and the slope of $\mathrm{pH}$ value was closed to Nernstian slope, indicating that the sensitivity increases with the loading of GO on the electrode. However, the peak current didn't increase when the GO concentration was beyond $0.15 \mathrm{mg} \cdot \mathrm{mL}^{-1}$, which could be attributed to the decrease of electrical conductivity due to excessive GO loaded on the electrode. Therefore, an optimum GO concentration of $0.15 \mathrm{mg} \cdot \mathrm{mL}^{-1}$ was selected for the fabrication of the cysteic acid/GO composite modified electrodes in this work.

The potentiometric responses of the $\mathrm{pH}$ sensors should also be concerned with the amount of cysteic acid adsorbed on the electrode. When the GO/GCE was swept by the cyclic voltammetry, the more cycles swept, the more cysteic acid molecules would be produced onto the surface of the GO/GCE electrode. Owing to more cysteic acid with more active groups, the potentiometric responses gradually increased with the sweep cycles performed on the electrode. But more cysteic acid would reduce the conductivity of the composite film. We finally chose 10 -sweep-circle under the cyclic voltammetry as optimized condition for electrochemical oxidation of L-cysteine in the present experiment.

\subsection{Calibration curve, response time and stability}

Under the optimum detection conditions, the emf response of the $\mathrm{pH}$ sensor based on cysteic acid/GO to $\mathrm{pH}$ changes was investigated using a Britton-Robinson (BR) buffer solution and performing simultaneous $\mathrm{pH}$ control employing a calibrated standard glass $\mathrm{pH}$ electrode dipped into the same solution. We found that the potentiometric response was proportional to the $\mathrm{pH}$ value in the range of 2.0 - 12.0 with a near-Nernstian slope of $50.2 \mathrm{mV} / \mathrm{pH}$ unit. Fig. 3 showed the linear relationship between $\mathrm{pH}$ values of testing solution and their corresponding potentials from which a linear equation can be established as: $E(\mathrm{~V})=$ $0.4215-0.0502(\mathrm{~V} / \mathrm{pH}) \cdot p H$. The correlation coefficient $\mathrm{R}^{2}=$ 0.997 can also be deduced.

The response time of the proposed electrode was determined from step changes of $\mathrm{pH}$ in BR buffer solution after injections of $\mathrm{HCl}$ or $\mathrm{NaOH}$ solutions. The response time was about $5 \mathrm{~s}$ at the $\mathrm{pH}$ of around 7.0. The response times of the $\mathrm{pH}$ sensor in solutions of different $\mathrm{pH}$ values were detected in the same way, and the results are in the

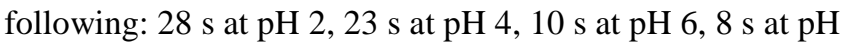
$8,21 \mathrm{~s}$ at $\mathrm{pH} 10,58 \mathrm{~s}$ at $\mathrm{pH} 12$. Therefore, the response times of the proposed $\mathrm{pH}$ sensor can be used for practical application.

The test stability of the $\mathrm{pH}$ sensor was carried out in a $0.1 \mathrm{~mol} \cdot \mathrm{L}^{-1}$ phosphate buffer solution of $\mathrm{pH} 5.0$ for 11 hours without interruption, at a temperature of $25^{\circ} \mathrm{C}$. The results demonstrated that the stability of the proposed $\mathrm{pH}$ sensor was excellent with a relative deviation standard of $0.80 \%$. The repeatability proved to be good when the $\mathrm{pH}$ sensor was repeatedly transferred between the solution of $\mathrm{pH} 4.0$ and $\mathrm{pH}$ 5.0, and the relative standard deviation is 
$0.51 \%$. The lifetime of the cysteic acid/GO/GC electrode had also been examined, and it demonstrated that the proposed electrode can retain $98.2 \%$ of its initial response after one-month-storage. This stability should be acceptable for most practical applications.

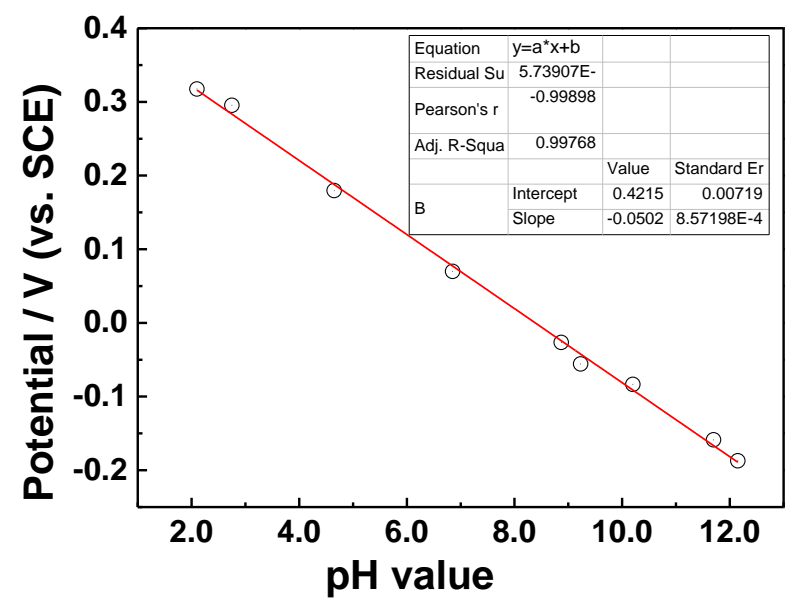

Fig. 3. Calibration curve of potentiometric response versus $\mathrm{pH}$ of cysteic acid/GO/GC electrode.

\subsection{Application of $\mathrm{pH}$ measurement in real samples}

The developed sensor for the $\mathrm{pH}$ detection was applied to test real samples including Coca Cola and tap-water, and then the result was shown in Table 1 . The relative deviations were $1.6 \%$ and $0.7 \%$, respectively. It can be seen that the proposed $\mathrm{pH}$ sensor can be used in detecting real aqueous samples.

Table 1. Detection of $\mathrm{pH}$ in real samples.

\begin{tabular}{|c|c|c|c|}
\hline Real samples & $\begin{array}{c}\text { Detected by } \\
\text { glass bulb } \\
\text { electrode }\end{array}$ & $\begin{array}{c}\text { Detected by } \\
\text { the proposed } \\
\mathrm{pH} \text { sensor }\end{array}$ & $\begin{array}{c}\text { Relative } \\
\text { deviation } \\
(\%)\end{array}$ \\
\hline Coca Cola & 3.87 & 3.81 & 1.6 \\
\hline Tap-water & 6.81 & 6.86 & 0.7 \\
\hline
\end{tabular}

\section{Conclusions}

We had developed a novel solid-state $\mathrm{pH}$ sensor based on the cysteic acid/GO composite film. The proposed $\mathrm{pH}$ sensor possessed a sensitivity of $50.2 \mathrm{mV} / \mathrm{pH}$, high stability (over a month) and a linear working range from $\mathrm{pH} 2$ to 12 at $25{ }^{\circ} \mathrm{C}$. The measurements of some real samples demonstrated that the proposed $\mathrm{pH}$ sensor is suitable for $\mathrm{pH}$ determination in real aqueous samples. The method also provides a potential to miniaturize $\mathrm{pH}$ sensor.

\section{Acknowledgment}

The work was supported by the National Natural Science Foundation of China (Grant Nos. 21106124, 21375116)

\section{References}

(1) Jeremy T. Robinson, F. Keith Perkins, Eric S. Snow, Zhongqing Wei, and Paul E. Sheehan: "Reduced graphene oxide molecular sensors", Nano Letters, Vol. 8, No. 10, pp. 3137-3140, 2008

(2) Helena Varela-Rizo, Ignacio Martin-Gullon, and Mauricio Terrones: "Hybrid film with Graphene Oxide and Metal Nanoparticles Could Now Replace Indium Tin Oxide", ACS nano, Vol. 6, No. 6, pp. 4565-4572, 2012

(3) Hua-Dong Huanga, Peng-Gang Renb, Jun Chena, Wei-Qin Zhanga, Xu Jia and Zhong-Ming Li: "High barrier graphene oxide nanosheet/poly(vinyl alcohol) nanocomposite films", Journal of Membrane Science, Vol. 409, pp. 156- 163, 2012

(4) Shaojun Guo and Shaojun Dong: "Graphene and its derivative-based sensing materials for analytical devices", Journal of Materials Chemistry, Vol. 21, pp. 18503-18516, 2011

(5) Teng Wang, Shengyi Zhang, Changjie Mao, Jiming Song, Helin Niu, Baokang Jin, Yupeng Tian: "Enhanced electrochemiluminescence of $\mathrm{CdSe}$ quantum dots composited with graphene oxide and chitosan for sensitive sensor", Biosensors and Bioelectronics, Vol. 31, No. 1, pp. 369-375, 2012

(6) Claire Berger, Zhimin Song, Tianbo Li, Xuebin Li, Asmerom Y. Ogbazghi, Rui Feng, Zhenting Dai, Alexei N. Marchenkov, Edward H. Conrad, Phillip N. First, and Walt A. de Heer: "Ultrathin epitaxial graphite: 2D electron gas properties and a route toward graphene-based nanoelectronics", The Journal of Physical Chemistry B, Vol. 108, No. 52, pp. 19912-19916, 2004

(7) Qi Guo and Xue Qin: "Flower-like $\mathrm{SnO}_{2}$ nanoparticles grown on graphene as anode materials for lithium-ion batteries", Journal of Solid State Electrochemistry, Vol. 18, No. 4, pp. 1031-1039, 2014

(8) Aming Wang, Hailong Wang, Shengyi Zhang, Changjie Mao, Jiming Song, Helin Niu, Baokang Jin, Yupeng Tian: "Controlled synthesis of nickel sulfide/graphene oxide nanocomposite for high-performance 
supercapacitor”, Applied Surface Science, Vol. 282, pp. 704-708, 2013

(9) Chengyin Wang, Zhixian Wang, Jun Guan and Xiaoya $\mathrm{Hu}$ : "Voltammetric Determination of Meloxicam in Pharmaceutical Formulation and Human Serum at Glassy Carbon Electrode Modified by Cysteic Acid Formed by Electrochemical Oxidation of L-cysteine", Sensors, Vol. 6, No. 9, pp. 1139-1152, 2006

(10) Winfried Vonau and Ulrich Guth: "pH monitoring: a review", Journal of Solid State Electrochemistry, Vol. 10, No. 9, pp. 746-752, 2006

(11) Roger G. Bates: "Determination of pH, Theory and Practice", New York, John Wiley \& Sons, Chap. 10., pp. 300-306, 1973

(12) Tae Yong Kim and Sung Yang: "Fabrication method and characterization of electrodeposited and heat-treated iridium oxide films for $\mathrm{pH}$ sensing", Sensors and Actuators B, Vol. 196, pp. 31-38, 2014

(13) Monika Glanc-Gostkiewicz, Marios Sophocleousa, John K. Atkinsona and Eduardo García-Breijo: "Performance of miniaturised thick-film solid state $\mathrm{pH}$ sensors", Sensors and Actuators A, Vol. 202, pp. 2-7, 2013

(14) Akira Yamada, Satoshi Mohri, Michihiro Nakamura and Keiji Naruse: "A fully automated $\mathrm{pH}$ measurement system for 96-well microplates using a semiconductor-based pH sensor", Sensors and Actuators B, Vol. 143, pp. 464-469, 2010

(15)Fei Xu , Guozheng Yan, Zhiwu Wang and Pingping Jiang: "Continuous accurate $\mathrm{pH}$ measurements of human GI tract using a digital pH-ISFET sensor inside a wireless capsule", Measurement, Vol. 64, pp. 49-56, 2015

(16) Jiao-Yang Li, Xu-Guang Huang, Wei Xu, Dong-Rui Xiao and Ze-Bing Zhong: "A fiber-optic $\mathrm{pH}$ sensor based on relative Fresnel reflection technique and biocompatible coating", Optical Fiber Technology, Vol. 20, pp. 28 - 31, 2014

(17)Zhi Li, Minghong Yang, Jixiang Dai, Gaopeng Wang, Chujia Huang, Jianguan Tang, Wenbin Hu, Han Song and Pengcheng Huang: "Optical fiber hydrogen sensor based on evaporated $\mathrm{Pt} / \mathrm{WO}_{3}$ film", Sensors and Actuators B, Vol. 206, pp. 564-569, 2015.

(18) Dmitriy A. Dikin, Sasha Stankovich, Eric J. Zimney, Richard D. Piner, Geoffrey H. B. Dommett, Guennadi Evmenenko, SonBinh T. Nguyen and Rodney S. Ruoff: "Preparation and characterization of graphene oxide paper”, Nature, Vol. 448, pp. 457-460, 2007
(19) T.R. Ralph: "The electrochemistry of L-cystine and L-cysteine: Part 1: Thermodynamic and kinetic studies", Journal of Electroanalytical Chemistry, Vol. 375, No. 1-2, pp. 1-15, 1994

(20) Lin Liu, Chengyin Wang and Guoxiu Wang: "Novel cysteic acid/reduced graphene oxide composite film modified electrode for the selective detection of trace silver ions in natural waters", Analytical Methods, Vol. 5, pp. 5812-5822, 2013

(21) Nicolae Spãtaru, Bulusu V. Sarada, Elena Popa, Donald A. Tryk, and Akira Fujishima: "Voltammetric determination of L-Cysteine at conductive diamond electrodes", Analytical Chemistry, Vol. 73, No. 3, pp. 514-519, 2001. 